\title{
Study On Machine Learning Algorithms
}

\section{Praba. R ${ }^{1}$, Darshan. G ${ }^{2}$, Roshanraj. K. T2, Surya Prakash. B}

${ }^{1}$ Assistant Professor, Department of Information Technology, Dr.N.G.P. Arts and Science College, Coimbatore, Tamil Nadu, India

${ }^{2}$ UG Candidate, Department of Information Technology, Dr.N.G.P. Arts and Science College, Coimbatore, Tamil Nadu, India

\begin{abstract}
Article Info

Volume 7, Issue 4

Page Number: 67-72

Publication Issue :

July-August-2021

Various machine learning algorithms are described in this work. These algorithms are used for a variety of applications, including data mining, image processing, predictive analytics, and so on. The fundamental benefit of employing machine learning is that once an algorithm learns what to do with data, it can complete tasks on its own.
\end{abstract}

Article History

Accepted : 02 July 2021

Published : 08 July 2021
Keywords : Machine Learning, Algorithms

\section{INTRODUCTION}

Machine learning is used to teach machines how to handle the data more efficiently. We may be unable to interpret the pattern or extract information from the data after examining it.In that case, we apply machine learning. With the abundance of datasets available, the demand for machine learning is in rise. Many industries from medicine to military apply machine learning to extract relevant information.

The phrase "Machine Learning" was coined by Arthur Samuel, a pioneer in the fields of artificial intelligence and computer games. Machine learning, he stated, is a "field of research that enables computers to learn without being explicitly programmed."
Machine Learning (ML) can be defined as the process of automating and refining the learning process of computers based on their experiences without the need for programming, i.e. without the use of humans. The process begins with providing highquality data, which is then used to train our machines (computers) by creating machine learning models based on the data and other methods. The algorithms we use are determined by the type of data we have and the task we are attempting to automate.

Machine learning (ML) is the study of computer algorithms that improve themselves over time as a result of experience and data. 1st It is consideredto be a component of artificial intelligence. Machine learning algorithms create a model based on sample

Copyright: @ the author(s), publisher and licensee Technoscience Academy. This is an open-access article distributed under the terms of the Creative Commons Attribution Non-Commercial License, which permits unrestricted non-commercial use, distribution, and reproduction in any medium, provided the original work is properly cited 
data, referred to as "training data," in order to make predictions or judgments without being explicitly programmed.

Machine learning algorithms are utilized in a wide range of applications, including medicine, email filtering, and computer vision, where developing traditional algorithms to do the required tasks is difficult or impossible.

However, not all machine learning is statistical learning. A subset of machine learning is strongly related to computational statistics, which focuses on making predictions using computers. The discipline of machine learning benefits from the study of mathematical optimization since it provides tools, theory, and application domains. Data mining is a similar branch of research that focuses on unsupervised learning for exploratory data analysis. Machine learning is also known as predictive analytics when it is used to solve business challenges.

\section{LITERATURE REVIEW}

Machine learning is the process of computers figuring out how to do things without being specifically programmed to do so. It entails computers learning from data in order to do specific jobs. It is possible to build algorithms that teach the computer how to perform all steps required to solve the problem at hand for basic jobs entrusted to computers; no learning is necessary on the computer's behalf. Manually creating the required algorithms for more complicated tasks can be difficult for a human. In practise, assisting the computer in developing its own algorithm rather than having human programmers explain each required step can prove to be more productive. Machine learning is a discipline that uses a variety of ways to train computers how to complete tasks for which no entirely suitable solution exists. When there are a large number of possible replies, one strategy is to classify some of the correct responses as valid. The computer can then utilize this as training data to refine the algorithm(s) it uses to determine right answers. The MNIST dataset of handwritten digits, for example, has frequently been used to train a system for the task of digital character recognition.

\section{TYPES OF LEARNING}

\section{A. Supervised Learning}

Supervised learning algorithms create a mathematical model of a set of data that includes both the inputs and the outputs that are sought a The information is referred to as training data, and it consists of a collection of training instances. Each training example has one or more inputs and a supervisory signal as the desired output. Each training sample is represented by an array or vector, sometimes referred to as a feature vector, and the training data is represented by a matrix in the mathematical model. Supervised learning techniques develop a function that may be used to predict the output associated with fresh inputs by iteratively optimizing an objective function. Active learning, classification, and regression are examples of supervised learning algorithms. When the outputs are limited to a small set of values, classification techniques are employed, and regression methods are used when the outputs can have any numerical value within a range. An incoming email, for example, would be the input to a classification algorithm that filters emails, and the output would be the name of the folder to file the email in. 


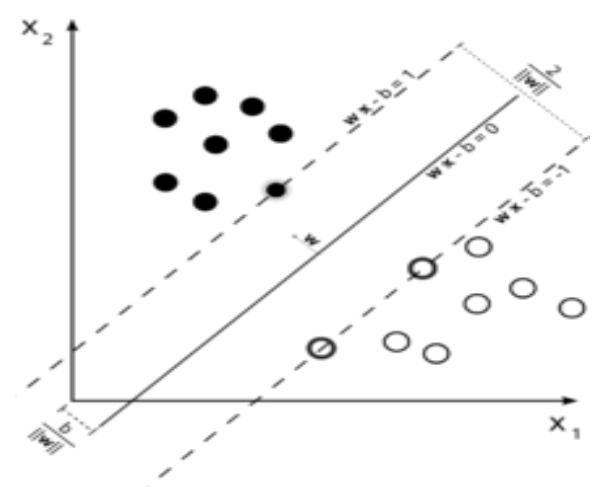

A supervised learning model called a support-vector machine separates data into areas separated by a linear border. The black circles are separated from the white circles by a linear barrier.

\section{B.Unsupervised Learning}

Unsupervised learning methods take a collection of data with only inputs and detect structure in it, such as data point grouping or clustering. As a result, the algorithms learn from unlabeled, unclassified, and uncategorized test data. Unsupervised learning algorithms discover commonalities in the data and react depending on the existence or lack of such commonalities in each new piece of data, rather than responding to feedback. The field of density estimation in statistics, such as calculating the probability density function, is a key application of unsupervised learning. Unsupervised learning, on the other hand, comprises various domains that need summarising and explaining data aspects.

Cluster analysis divides a set of observations into subsets (called clusters) so that observations within the same cluster are comparable based on one or more predetermined criteria, while observations from different clusters are distinct. Different clustering approaches make different assumptions about the structure of the data, which is commonly characterized by some similarity metric and evaluated, for example, by internal compactness, or the similarity between cluster members, and separation, or the difference between clusters.
Estimated density and graph connectedness are used in other approaches.

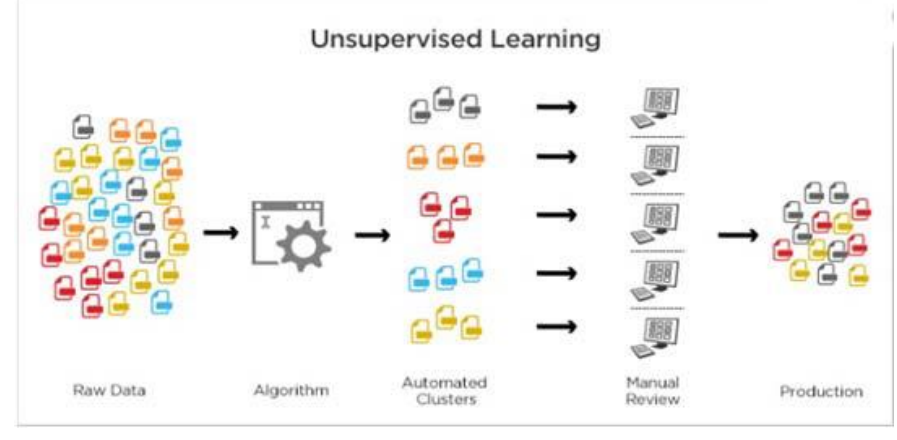

C. Semi-supervised Learning

Unsupervised learning (without any labelled training data) and supervised learning (with labelled training data) are the two types of learning (with completely labelled training data). Although some of the training examples lack training labels, several machine-learning researchers have discovered that unlabeled data, when combined with a modest amount of labelled data, can enhance learning accuracy significantly. The training labels in weakly supervised learning are noisy, limited, or imprecise, yet they are generally cheaper to obtain, resulting in larger effective training sets.

\section{Reinforcement learning}

Reinforcement learning is a branch of machine learning that studies how software agents should behave in a given environment in order to maximize some metric of cumulative reward. Game theory, control theory, operations research, information theory, simulation-based optimization, multi-agent systems, swarm intelligence, statistics, and genetic algorithms are among the numerous disciplines that study the field due to its generality. The environment is generally represented as a Markov decision process in machine learning (MDP). Dynamic programming techniques are used in many reinforcement learning systems. When exact mathematical models of the MDP are infeasible, reinforcement learning procedures are applied. Reinforcement learning 
algorithms are employed in autonomous vehicles and in teaching humans how to play a game.

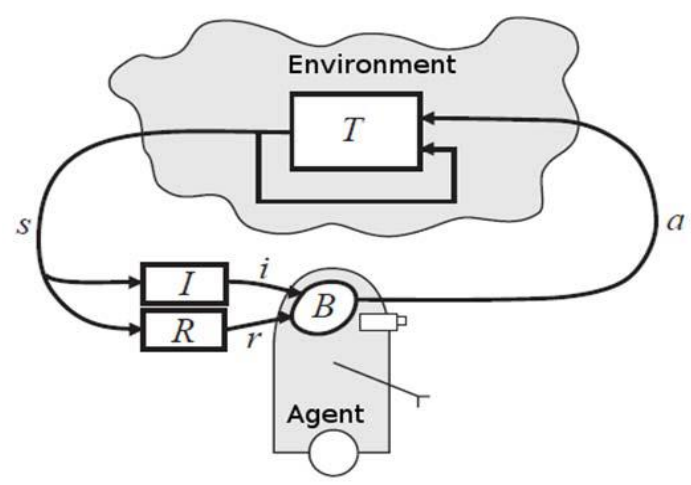

E. Dimensionality Reduction

The technique of lowering the number of random variables under consideration by generating a set of primary variables is known as dimensionality reduction. In other words, it's a method of minimising the size of your feature set, also known as the number of features. The majority of dimensionality reduction approaches fall into one of two categories: feature deletion or extraction. Principal component analysis is one of the most widely used methods for dimensionality reduction.

\section{Analyze the Principal Components (PCA)}

PCA entails converting higher-dimensional data (such as 3D) to a smaller space (eg. 2D). This results in a decreased data dimension (2D rather than 3D), while preserving all of the original variables in the model and not modifying the data.

A supervised learning model called a support-vector machine separates data into areas separated by a linear border. The black circles are separated from the white circles by a linear barrier.

\section{MODELS}

\section{Artificial neural networks}

Artificial neural networks (ANNs), also known as connectionist systems, are computing systems that are based on biological neural networks found in animal brains. Such systems "learn" to execute tasks by considering examples, usually without any taskspecific rules being coded. An artificial neural network (ANN) is a model built on a set of connected units or nodes known as "artificial neurons," which are roughly modelled after the neurons in a biological brain. Each link, like the synapses in a human brain, can send information, or a "signal," from one artificial neuron to the next.

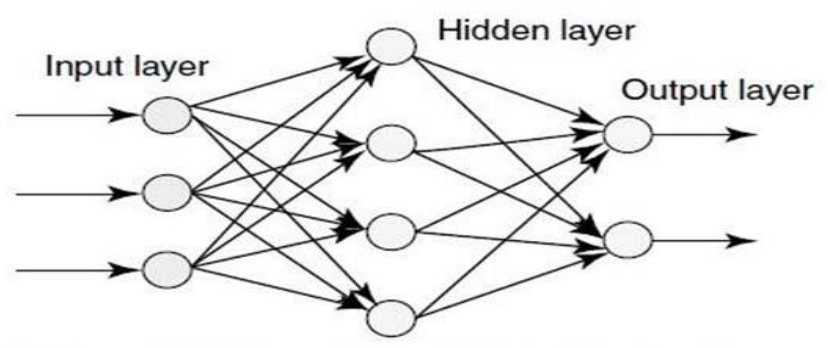

\section{Decision Tree}

To get from observations about an item (represented in the branches) to conclusions about the item's goal value, decision tree learning employs a decision tree as a predictive model (represented in the leaves). In statistics, data mining, and machine learning, it is one of the predictive modelling methodologies. Classification trees are tree models in which the goal variable can take a discrete set of values; in these tree structures, leaves indicate class labels and branches represent feature combinations that lead to those class labels.

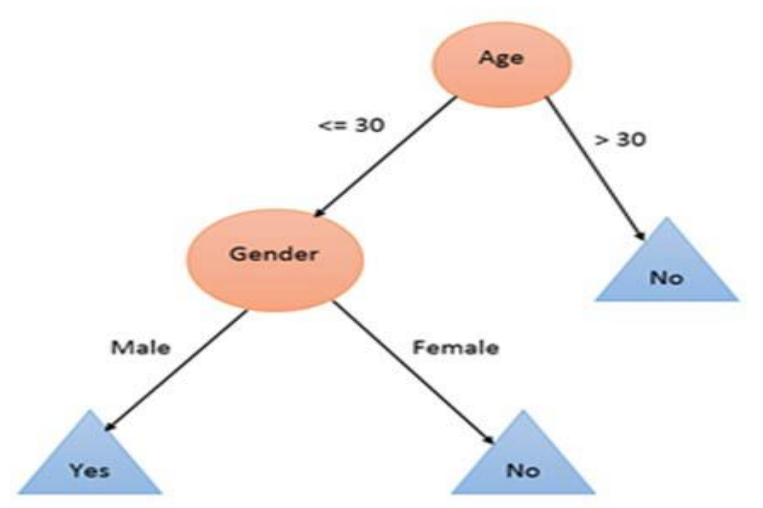

\section{Support-Vector Networks}

SVMs, also known as support-vector networks, are a group of similar supervised learning algorithms for 
classification and regression. An SVM training method creates a model that predicts whether a new example falls into one of two categories given a set of training examples that are individually labelled as belonging to one of two categories. Although methods such as Platt scaling exist to employ SVM in a probabilistic classification environment, an SVM training algorithm is a non-probabilistic, binary, linear classifier. SVMs may perform non-linear classification as well as linear classification by implicitly mapping their inputs into highdimensional feature spaces, which is known as the kernel trick.

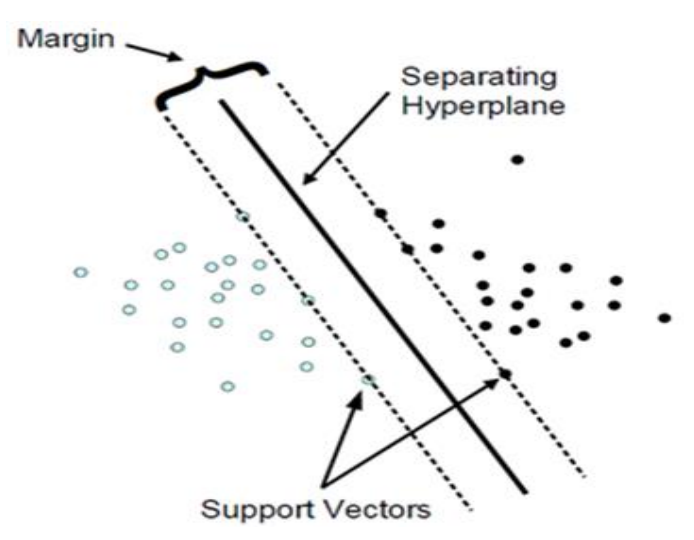

\section{Regression analysis}

Regression analysis is a broad term that refers to a number of statistical techniques for estimating the relationship between input variables and their associated characteristics. Linear regression is the most frequent type, in which a single line is generated to best match the available data using a mathematical criterion such ordinary least squares.

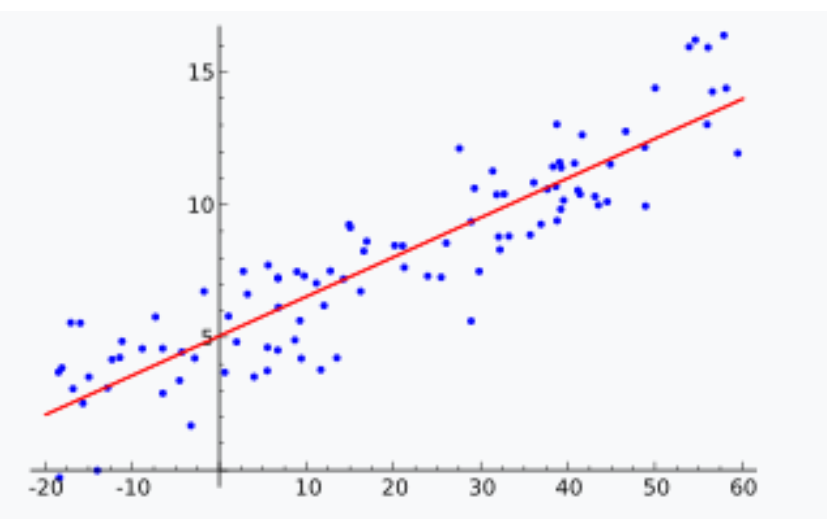

5. Bayesian network

A Bayesian network, also known as a belief network or a directed acyclic graphical model, is a probabilistic graphical model that uses a directed acyclic graph to describe a set of random variables and their conditional independence (DAG). A Bayesian network, for example, could be used to illustrate the probability correlations between diseases and symptoms. The network may be used to calculate the chances of certain diseases being present based on symptoms. There are efficient algorithms for inference and learning. Dynamic Bayesian networks are Bayesian networks that model sequences of variables, such as speech signals or protein sequences.

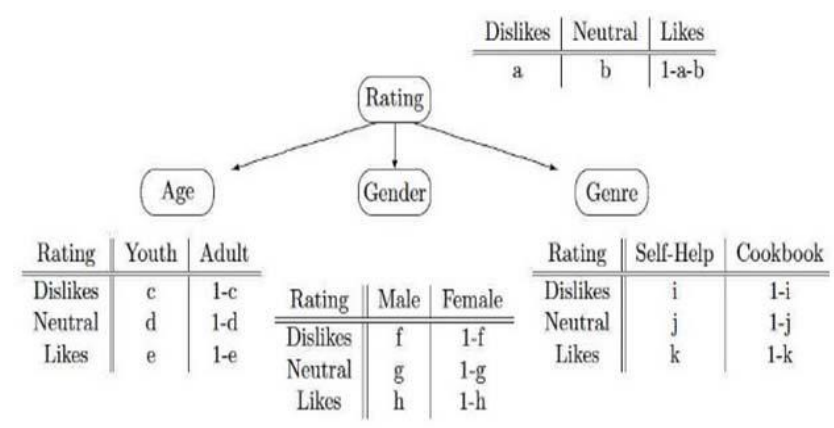

\section{CONCLUSION}

This study examines a number of different machine learning algorithms. Today, everyone, intentionally or unconsciously, employs machine learning. From online shopping for a recommended product to uploading images on social networking sites, there's a lot to do. This document provides an overview of the most widely used machine learning algorithms.

\section{REFERENCES}

[1]. R. Praba, “A Study on Data Science Basics with Python Concepts", Volume-4, Issue-14, ISSN: 2582-3930. 
[2]. W. Richert, L. P. Coelho, "Building Machine Learning Systems with Python", Packt Publishing Ltd., ISBN 978-1-78216-140-0

[3]. M. Welling, "A First Encounter with MachineLearning"

[4]. M. Bowles, "Machine Learning in Python: Essential Techniques for Predictive Analytics", John Wiley \& Sons Inc., ISBN: 978-1-11896174-2

[5]. S.B. Kotsiantis, "Supervised Machine Learning: A Review of Classification Techniques", Informatica 31 (2007) 249-268

[6]. L. Rokach, O. Maimon, “Top - Down Induction of Decision Trees Classifiers - A Survey", IEEE Transactions on Systems,

[7]. D. Lowd, P. Domingos, "Naïve Bayes Models for Probability Estimation"

[8]. https://webdocs.cs.ualberta.ca/ greiner/C651/Homework2_Fall2008.html

[9]. D. Meyer, "Support Vector Machines - The Interface to libsvm in package e1071", August 2015

[10]. S. S. Shwartz, Y. Singer, N. Srebro, "Pegasos: Primal Estimated sub - Gradient Solver for SVM", Proceedings of the 24th InternationalConference on Machine Learning, Corvallis, OR, 2007

[11]. http://www.simplilearn.com/what-is-machinelearning-and-why-itmatters- article

[12]. P. Harrington, "Machine Learning in action", Manning Publications Co., Shelter Island, New York, 2012

\section{Cite this article as :}

Praba. R, Darshan. G, Roshanraj. K. T, Surya Prakash. B, "Study On Machine Learning Algorithms", International Journal of Scientific Research in Computer Science, Engineering and Information Technology (IJSRCSEIT), ISSN : 2456-3307, Volume 7 Issue 4, pp. 67-72, July-August 2021. Available at doi : https://doi.org/10.32628/CSEIT2173105

Journal URL : https://ijsrcseit.com/CSEIT2173105 